\section{MS34-P4 Solubility-based solvate screening of pharmaceutical substance trospium chloride}

Veronika Sládková ${ }^{1}$, Tereza Skalická ${ }^{1}$, Eliška Skořepová ${ }^{1}$, Jan Čejka ${ }^{1}, V^{2}$ clav Eigner ${ }^{1,2}$, Bohumil Kratochvíl $^{1}$

1. Department of Solid State Chemistry, University of Chemistry and Technology, Prague, Technicka 5, 16628, Prague 6, Czech Republic

2. Institute of Physics AS CR, v.v.i., Na Slovance 2, 18221 Prague 8, Czech Republic

email: sladkovv@vscht.cz

The ability of long-established pharmaceutical compound trospium chloride ( $\mathrm{TCl})$ to form solvates was investigated. Taking into account the solubility of $\mathrm{TCl}$ (and, therefore, material consumption), different solvate screening approaches were considered: slow evaporation, slurrying and anti-solvent addition. We applied them on 20 polar solvents. Five solvates, with solvents methanol, acetonitrile, propionitrile, $N, N$-dimethylformamide, nitromethane and a dihydrate were identified and characterized by various analytical techniques. The structures of all solvates were determined by single-crystal X-ray diffraction. The reproducible forms were further characterized by powder X-ray diffraction, desolvation behaviour was observed by thermoanalytical (TGA/DSC) methods and solubility in particular solvents was determined via UV-Vis spectrophotometry or gravimetric measurements. Structural features of novel solvates and of previously described polymorphs and cocrystals of $\mathrm{TCl}$ were compared, presented by a tree diagram which classifies the structures according to their molecular packing.

This work was supported by the Grant Agency of Czech Republic, Grant no. 106/14/03636S and received financial support from specific university research (MSMT No 20/2015).

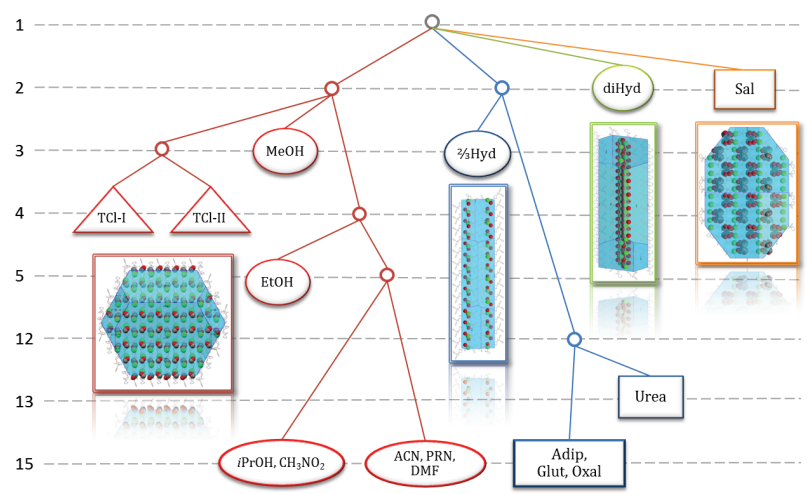

Figure 1. Tree diagram of trospium chloride solid forms

Keywords: pharmaceuticals, solvates, screening, X-ray crystallography

\section{MS34-P5 Molecular dynamics of supersaturated indometacin-nicotinamide solutions}

László Fábián ${ }^{1}$, Karol P. Nartowski ${ }^{1}$, Yaroslav Z. Khimyak ${ }^{1}$

1. School of Pharmacy, University of East Anglia, Norwich, UK

email: 1.fabian@uea.ac.uk

Recently, co-crystallisation has been shown to allow the growth of single crystals from compounds that could not be crystallised on their own [1]. This observation directed our attention towards the role of solution-state supramolecular assemblies in the formation of cocrystals. A relatively simple and experimentally well-characterised system, the indometacin-nicotinamide co-crystal [2] dissolved in alcohols or acetonitrile was selected for preliminary investigations.

The formation and stability of molecular aggregates were studied through a series of molecular dynamics simulations. As starting points, pre-aligned supramolecular synthons from the crystal structure or randomly placed solutes in cubic solvent boxes were used. The behaviours of dilute, saturated and supersaturated solutions were compared. Trajectories were simulated and analysed using the Gromacs package [3] and the general amber force field [4].

The results show that simple hydrogen-bonded dimers are not stable in these solutions, but pairs of molecules often remain in close proximity even when there are no longer hydrogen bonds between them. Indometacin-indometacin and indometacin-nicotinamide contacts are more persistent than nicotinamide-nicotinamide contacts. In both saturated and the supersaturated solutions, a dynamic set of clusters is formed, with the mean cluster size being bigger in the supersaturated solutions. This result is qualitatively consistent with the observed gradual decrease of the diffusion coefficients with increasing concentration, which was measured by solution PFG NMR spectroscopy. Further changes in the ${ }^{1} \mathrm{H}$ chemical shifts of the dissolved species with increasing solute concentration indicate changes in the local environment of the aggregating molecules. The clusters are dynamic both in terms of their size and structure, i.e., no crystal-like aggregates are observed. Both the mean and maximum cluster size show a marked fluctuation, but no appreciable drift towards larger sizes.

1. K. S. Eccles, R. E. Deasy, L. Fábián, A. R. Maguire and S. E. Lawrence, J. Org. Chem., 2011, 76, 1159.

2. A. Alhalaweh, A. Sokolowski, N. Rodríguez-Hornedo and S. P. Velaga, Cryst. Growth. Des., 2011, 11, 3923.

3. B. Hess, C. Kutzner, D. van der Spoel and E. Lindahl, J. Chem. Theory Comput., 2008, 4, 435.

4. J. Wang, R. M. Wolf, J. W. Caldwell, P. A. Kollman and D. A. Case, J. Comput. Chem., 2004, 25, 1157.

Keywords: cocrystal; molecular dynamics; pre-nucleation assemblies 